\title{
Integration of nondegradable polystyrene and degradable gelatin in a core-sheath nanofibrous patch for pelvic reconstruction
}

This article was published in the following Dove Press journal:

International Journal of Nanomedicine

24 April 2015

Number of times this article has been viewed

\author{
Liangpeng $\mathrm{Ge}^{1-5, *}$ \\ Qingtao $\mathrm{Li}^{2,3, *}$ \\ Junzi Jiang 2,3 \\ Xiaoyan You' \\ Zuohua Liu' \\ Wen Zhong ${ }^{6}$ \\ Yong Huang' \\ Malcolm MQ Xing ${ }^{2,3}$ \\ 'Chongqing Academy of Animal \\ Sciences, Chongqing, People's Republic \\ of China; ${ }^{2}$ Department of Mechanical \\ and Manufacturing Engineering, \\ Biochemistry and Medical Genetics, \\ University of Manitoba, Winnipeg, MB, \\ Canada; ${ }^{3}$ Manitoba Institute of Child \\ Health, Winnipeg, MB, Canada; ${ }^{4} \mathrm{Key}$ \\ Laboratory of Pig Industry Sciences, \\ Ministry of Agriculture, Chongqing, \\ People's Republic of China; \\ ${ }^{5}$ Chongqing Key Laboratory of Pig \\ Industry Sciences, Chongqing, People's \\ Republic of China; 'Department of \\ Textile Sciences, Faculty of Human \\ Ecology, University of Manitoba, \\ Winnipeg, MB, Canada \\ *These authors contributed equally \\ to this work
}

Correspondence: Malcolm MQ Xing

Department of Mechanical and

Manufacturing Engineering,

Biochemistry and Medical Genetics,

University of Manitoba, 75A Chancellors

Circle, University of Manitoba, MB R3T

2N2, Canada

Email malcolm.xing@umanitoba.ca

Yong Huang

Chongqing Academy of Animal

Sciences, No.770, Changzhou Road,

Rongchang, Chongqing, 402460,

People's Republic of China

Email329439047@qq.com

\begin{abstract}
Pelvic organ prolapse (POP) is a serious health issue affecting many adult women. Complications of POP include pelvic pressure, pelvic pain, and problems in emptying their bowels or bladder. Sometimes, POP may even cause urinary outflow obstruction and lead to bladder or kidney infections. Currently, synthetic and naturally derived materials have been chosen for treatment of POP to reduce the high recurrence rates after surgical interventions. However, existing materials for POP treatment cannot meet the clinical requirements in terms of biocompatibility, mechanics, and minimal risk of rejection. Especially, erosion in synthetic polymers and rapid degradation in natural polymers limit their further applications in clinics. To address these concerns, we report a novel POP replacement using core-sheath polystyrene/ gelatin electrospun nanofiber mesh. The outside gelatin sheath provides a hydrophilic surface and implantable integrity between host and guest, while the inner PS core offers the necessary mechanical support. The composite mesh shows graft accommodation in pelvic submucosa after implantation in vivo, as shown in hematoxylin-eosin staining and T helper cell phenotype and macrophage phenotype stainings. Qualitative analysis of inducible nitric oxide synthase, arginase, interferon- $\gamma$, and interleukin-10 gene expressions also indicates that the implanted composite mesh switches to accommodation mode 2 weeks postimplantation. Thus, these novel core-sheath polystyrene/gelatin nanofibrous membranes are promising in pelvic reconstruction.
\end{abstract}

Keywords: core-sheath nanofibrous patch, polystyrene, gelatin, pelvic reconstruction

\section{Introduction}

Pelvic organ prolapse (POP) is a major health issue and occurs in 50\% adult women. ${ }^{1,2}$ Among them, around 11\% require prolapse surgery, and one third need several operations because of recurrent prolapse. ${ }^{3,4}$ To provide support to the weakened area and lower the high recurrence rate, treating POP by implanting biomaterials is a common strategy of surgical intervention. ${ }^{5,6}$ Synthetic and naturally derived materials vary considerably in their properties for application in clinical POP treatment. Synthetic materials used for POP treatment include polyethylene tetraphthalate mesh, polypropylene (PP) mesh, and polytetrafluoroethylene. Naturally derived materials for POP treatment are cadaveric human fascia lata, dura, or dermis; porcine dermis; and porcine small intestine submucosa (SIS). ${ }^{7,8}$ PP mesh is a widely used nondegradable synthetic mat that is long-lasting, inert, nontoxic, and antigenic. Clinical tests, however, show dyspareunia, infection, and erosion or extrusion in PP mats. ${ }^{9,10}$ For example, a high vaginal mesh erosion rate $(15.6 \%)$ at 3 months after surgery was reported. ${ }^{11,12}$ On the other hand, naturally derived materials such as porcine dermis and SIS have many ideal characteristics for POP replacement, including cell compatibility and degradable properties. Their structural and functional proteins are similar to the native mammalian extracellular 
matrix, providing a potential "seamless" interaction between host and implant. ${ }^{13}$ They can also reduce the complications of erosion and fistula formation. ${ }^{14,15}$ However, most natural polymers are not durable because of their high degradation rate. They are also not recommended for high-strain environments in pelvic reconstruction as they usually fail to offer sufficient mechanical support..$^{13,15-17}$ For example, although porcine SIS can improve quality-of-life outcomes by attenuating prolapse severity for 6 months, there was no significant difference in improving role limitations, physical limitations, and emotions, compared to traditional anterior repair, after 2 years. ${ }^{14,18}$ Hybrid materials are undoubtedly the ideal choice to meet the demands for POP reconstruction because strength and durability of the synthetic component can be combined with the biocompatibility of the biologic materials. Therefore, it will be an ideal substitute for pelvic floor repair if longlasting synthetic, as well as cell-compatible and attachable natural, polymers are included to meet the physicochemical and biological requirements for POP reconstruction.

To this end, we developed a core (polystyrene, PS)-sheath (gelatin) nanofibrous mat for POP reconstruction (Figure 1). We hypothesize that the PS core in nanofibers enhances the mat's durability and mechanical strength, and the gelatin sheath contributes to good compatibility and cell adhesion. It can be an ideal patch for pelvic floor repair based on the following three facts. First, PS is widely used in cell culture applications because of its strength, nontoxicity, heat stability, and slow degradation. Second, gelatin has long been known to provide good cell adhesion, and gelatin coating on PS surface has been a routine approach to increasing cell adhesion and proliferation. ${ }^{19}$ Third, PS and gelatin are both US Food and Drug Administration (FDA)-approved polymers suitable for clinics. In this study, we first tested the physical characteristics

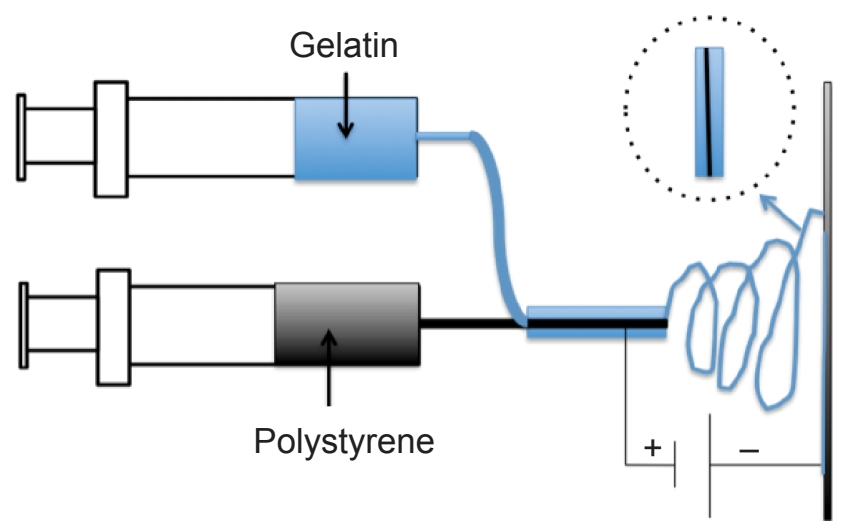

Figure I The schematic diagram of electrospinning setup for core-sheath PS/gelatin nanofibrous membrane

Abbreviation: PS, polystyrene. of PS/gelatin nanofibrous mat and then investigated the cell adhesion. Finally, the membranes were implanted under pelvic submucosa in Sprague-Dawley rats, and the host's responses to the PS/gelatin nanofibrous mats were monitored to verify the biocompatibility of the membrane.

\section{Experimental section Materials}

PS was purchased from Scientific Polymer Inc (ON, Canada). Acetic acid and ethyl acetate were purchased from Fisher Scientific (Toronto, ON, Canada). Gelatin (from porcine skin, Type A), dimethylformamide (DMF), penicillin, and streptomycin were all purchased from Sigma-Aldrich without further purification. Dulbecco's Modified Eagle's Medium (DMEM), fetal bovine serum (FBS), Dulbecco's phosphate-buffered saline (no calcium, no magnesium) (D-PBS), trypsin-ethylenediaminetetraacetic acid, were all purchased from Invitrogen (Burlington, ON, Canada). C57BL/6 Mouse adipose-derived mesenchymal stem cells with green fluorescent protein (GFP) (ADMSCs-GFP) were purchased from American Type Culture Collection.

\section{Preparation of PS/gelatin nanofibrous membrane}

Gelatin (2 g) was dissolved in $10 \mathrm{~mL}$ of mixture containing acetic acid, distilled water, and ethyl acetate (volume ratio $=6: 3: 1$, with stirring overnight at room temperature. Meanwhile, 2 g PS was dissolved at $10 \mathrm{~mL} \mathrm{DMF}$, stirring overnight at room temperature. The schematic setup of coaxial electrospinning used in this study is as follows: gelatin solution and PS solution were placed in two syringes mounted in a syringe pump (PHD2000 Infusion). The inner needle (for PS solution) had a diameter of $0.4 \mathrm{~mm}$ and the outer needle's (for gelatin solution) diameter was $0.8 \mathrm{~mm}$. Electrospinning was accomplished using a high-voltage direct current power supply (Gamma High Voltage Research Inc) set to $20 \mathrm{kV}$, a $14 \mathrm{~cm}$ tip-to-substrate distance, and a $1 \mathrm{~mL} / \mathrm{h}$ flow rate. A $25 \times 25 \mathrm{~cm}$ sheet approximately $100 \mu \mathrm{m}$ in thickness was deposited onto aluminum foil. The PS/gelatin sheets were exposed to glutaraldehyde vapor for 3 hours and then placed in a vacuum overnight to ensure removal of residual glutaraldehyde.

\section{Scanning electron microscopy}

The surface morphology of the PS/gelatin nanofibrous membrane was observed under a scanning electron microscope (JSM-5900LV; JEOL) at an accelerating voltage of $20 \mathrm{kV}$. 


\section{Contact angle measurement}

The surface contact angles were measured using Drop Shape Analysis System (DSA100; KRUSS, Germany). Deionized water was dropped onto the sample from a needle on a microsyringe, and contact angles were calculated based on the shape of the drop. In our study, the contact angle $\theta$ was derived as an average of ten measurements.

\section{Mechanical characterization}

To evaluate the mechanical properties of the PS/gelatin nanofibrous membrane, dog-bone-shaped samples with width $5 \mathrm{~mm}$ and gage length $20 \mathrm{~mm}$ were prepared, and the thickness of the samples was around $0.1 \mathrm{~mm}$. The tensile properties were determined utilizing a $5 \mathrm{~N}$ load cell with a strain rate of $5 \mathrm{~mm} / \mathrm{min}$ on universal tensile tester (INSTRON 5965), and the Young's modulus of the patches was given by the instrument's software.

\section{Cell culture}

Mouse ADMSCs-GFP was utilized to assess the biocompatibility of the nanofibrous membrane. Cells were cultured in DMEM supplemented with $10 \%$ FBS, 100 units $/ \mathrm{mL}$ penicillin, and $100 \mu \mathrm{g} / \mathrm{mL}$ streptomycin at $37^{\circ} \mathrm{C}$ in $5 \% \mathrm{CO}_{2}$. Nanofibrous membranes (diameter $=6 \mathrm{~mm}$ ) were sterilized with $70 \%$ ethanol solutions for 30 minutes, rinsed three times in PBS to remove any residual ethanol, and partially dried using sterile absorbent tissue paper. Subsequently, $2 \times 10^{4}$ cells $/ \mathrm{cm}^{2}$ were seeded on the surface of membranes and incubated for 2 hours to allow cells to become effectively attached on the scaffold surface; ${ }^{20-22}$ then fresh medium was added for continuing the culture. After 24 hours of incubation, the membranes were washed with PBS and examined by fluorescence microscopy.

\section{In vivo scaffold implantation}

Twelve 12-week-old Sprague-Dawley rats were housed individually in shoebox cages. They were handled in a laminar flow hood following aseptic techniques. All procedures were performed in accordance with National Institutes of Health guidelines and with the approval of the Institutional Animal Care and Use Committee at the Chongqing Academy of Animal Sciences. Each rat was anesthetized with 3\% pentobarbital injection into the peritoneal cavity at a dose of $0.1 \mathrm{~g} / \mathrm{kg}$. The surgical site of the pudendum was prepared by removal of any fur and sterilized using a Betadine solution (povidone iodine; Sichuan Kelun Co, Ltd, Chengdu, People's Republic of China), followed by placement of sterile drapes. A $1 \mathrm{~cm}$ incision was made in the vaginal mucosa, and a square of $1 \mathrm{~cm} \times 1 \mathrm{~cm}$ was created in the exposed cavity, leaving the underlying submucosa. The area was then implanted with one of the test materials. Chlortetracycline was painted on the wound postoperatively. The animals were monitored daily for signs of infection or inflammation at the surgical site. All animals survived the surgical procedure and their predetermined study period without complications. ${ }^{16}$

Animals were sacrificed at 1 week, 2 weeks, 3 weeks, 4 weeks, and 6 weeks postsurgery. Each rat was euthanized with $0.5 \mathrm{~mL}$ of $3 \%$ pentobarbital injected into the peritoneal cavity, followed by an intracardiac injection of $5 \mathrm{~mL}$ of potassium chloride to induce cardiac arrest. The grafts were explanted with a small amount (approximately $2 \mathrm{~mm}$ ) of the surrounding native tissue. One half of the tissue was fixed in $10 \%$ neutral buffered formalin and then embedded in paraffin. The other half of each specimen was preserved in RNAlater ${ }^{\mathrm{TM}}$ (Ambion, Austin, TX, USA) for RNA extraction.

\section{Histologic and immunohistochemical staining}

Specimens at the explanted wound site were fixed in formalin and embedded in paraffin before being cut into $6 \mu \mathrm{m}$ thick sections and mounted on glass slides. The specimens were deparaffinized with xylene, followed by exposure to a series of gradient ethanol solutions (100\%-70\%). Sections were subjected to hematoxylin and eosin (H\&E) staining for morphological assessment. Immunohistochemistry was performed on consecutive $6 \mu \mathrm{m}$ sections of each specimen in order to investigate cell phenotype within and surrounding the implant wound site. The slides were stained by an avidin-biotin method as described previously. ${ }^{16}$ The primary antibodies used here were rabbit anti-rat CXCR3 (T helper cell 1 [Th1] phenotype marker), rabbit anti-rat CCR4 (Th2 phenotype marker), rabbit anti-rat CCR7 (M1 phenotype macrophage marker), and rabbit anti-rat CD163 (M2 phenotype macrophage marker), at a dilution of 1:100. The secondary antibody used in this study was goat antirabbit immunoglobulin $\mathrm{G}(\operatorname{IgG})$, at a dilution of 1:100. All antibodies were purchased from the Beijing Biosynthesis Biotechnology Co, Ltd, and were diluted in filtered PBS ( $\mathrm{pH}$ 7.4) before use.

A qualitative analysis of the histological morphology of each specimen was performed as described previously. ${ }^{23} \mathrm{~A}$ ratio of the percentage of M1 to M2 cells and Th1 to Th2 cells were calculated as follows, respectively:

M1:M2 = percentage of M1 cells/percentage of M2 cells Th1:Th2 $=$ percentage of Th1 cells/percentage of Th2 cells 
Values were plotted on a log scale such that values $>1.0$ were representative of the predominance of M1 cells or Th1 cells among those cells. Conversely, a value of $<1.0$ was representative of a predominance of M2 cells or Th2 cells.

\section{Gene expression analysis}

Total RNA isolation and cDNA synthesis were conducted from explanted tissues as described previously. ${ }^{23}$ Then quantitative real-time polymerase chain reaction was performed by the SYBER Green method (Applied Biosystems, Thermo Fisher Scientific, Waltham, MA, USA). The reactants were denatured at $95^{\circ} \mathrm{C}$ for 10 minutes, then subjected to 40 cycles of $95^{\circ} \mathrm{C}$ for 15 seconds and $60^{\circ} \mathrm{C}$ for 1 minute. The mRNA expression was normalized to that of the housekeeping gene glyceraldehyde 3-phosphate dehydrogenase for each sample ( $n=3$ per group). ${ }^{24}$ Sequences of the primers synthesized were as shown in Table 1.

\section{Statistical analysis}

Significant differences between two groups were evaluated using a one-way analysis of variance with a 95\% confidence interval, and $P<0.05$ was considered statistically significant.

\section{Results and discussion}

An ideal patch for POP treatment should have a desired balance among its physical, chemical, and biological properties. ${ }^{25}$ However, existing synthetic and natural materials for POP treatment in clinics cannot fully meet these requirements, as shown by 1) the high dyspareunia, infection, and erosion or extrusion rate resulting from the synthesis scaffolds and 2) the rapid degradation of natural scaffolds. ${ }^{9-16}$ To overcome these problems, we constructed a composite nanofibrous membrane by electrospinning nanofibers with a PS core embedded in a gelatin sheath as a potential POP. Electrospinning provides a facile approach to fabricate nanofibrous scaffolds similar to natural extracellular matrix, in that they have large surface volume ratio for three-dimensional cell ingrowth and controlled release of bioactive molecules. ${ }^{26-28}$ We hypothesized that the PS/gelatin nanofibrous patch can integrate the advantages of synthetic and natural biologic materials for pelvic reconstruction, although this device is not designed for human use yet at this stage.

\section{Synthesis and characterization of PS/gelatin nanofibrous mats}

In order to form a core (PS)-sheath (gelatin) nanofibrous mat for POP reconstruction, 20\% gelatin solution and 20\% PS solution were used to produce the shell and core of nanofibers using coaxial electrospinning. PS nanofibers and gelatin nanofibers were also produced as controls. Scanning electron microscopy results (Figure 2) show that all samples (PS, gelatin, PS/gelatin nanofibrous membrane) showed a three-dimensional reticulate nanostructure (Figure $2 \mathrm{~A}-\mathrm{C}$ ). To verify that gelatin was coated on the surface of the PS fiber core, fluorescein isothiocyanate-conjugated gelatin was coaxially electrospun with PS, and the confocal fluorescence image (Figure 2D) suggests that gelatin was uniformly distributed on the sheath.

The successful coating of gelatin was also further confirmed using attenuated total reflection-infrared spectroscopy (ATR-IR) mapping. Figure 3 shows the Fourier transform infrared spectroscopy (FTIR-ATR) spectra of gelatin, PS, and PS/gelatin. The observed IR spectra of gelatin and PS are in agreement with previous reported results. The gelatin showed the most characteristic peaks at $3,300.26 \mathrm{~cm}^{-1}$ corresponding to the amide A, $2,923.48 \mathrm{~cm}^{-1}$ to the amide $\mathrm{B}, 1,650.66 \mathrm{~cm}^{-1}$ to the amide I, $1,539.84 \mathrm{~cm}^{-1}$ to the amide II, and $1,245.38 \mathrm{~cm}^{-1}$ to the amide III. In the region of the FTIR spectrum of PS, the peaks at $3,027.97 \mathrm{~cm}^{-1}$ and $2,913.98 \mathrm{~cm}^{-1}$ are due to the $\mathrm{sp} 2 \mathrm{C}-\mathrm{H}$ and sp3 C-H stretching, respectively. The peaks at $1,600.00 \mathrm{~cm}^{-1}$ and $1,457.52 \mathrm{~cm}^{-1}$ represent the bonding stretching vibration of sp2 $\mathrm{C}=\mathrm{C}$ and the bending vibration of bonding sp3 $\mathrm{C}-\mathrm{H}$, respectively. The bands with peak locations at $760.95 \mathrm{~cm}^{-1}$ and $697.63 \mathrm{~cm}^{-1}$ are caused by vibration of phenyl wagging and twisting, respectively. As expected, the FTIR-ATR spectrum

Table I Primer sequences for qRT-PCR analysis

\begin{tabular}{lll}
\hline Gene & Forward primer $\left(\mathbf{5}^{\prime} \mathbf{-} \mathbf{3}^{\prime}\right)$ & Reverse primer $\left(\mathbf{5}^{\prime} \mathbf{- 3}\right)$ \\
\hline iNOS & GAGACGCACAGGCAGAGG & CAGGCACACGCAATGATGG \\
ARG & CATATCTGCCAAGGACATCG & GGTCTCTTCCATCACTTTGC \\
IFN- $\gamma$ & CACGCCGCGTCTTGGT & TCTAGGCTTTCAATGAGTGTGCC \\
IL-I0 & GTTGCCAAGCCTTGTCAGAAA & TTTCTGGGCCATGGTTCTCT \\
GAPDH & GGCAAGTTCAATGGCACAGT & TGGTGAAGACGCCAGTAGACTC \\
\hline
\end{tabular}

Abbreviation: qRT-PCR, quantitative real-time polymerase chain reaction. 

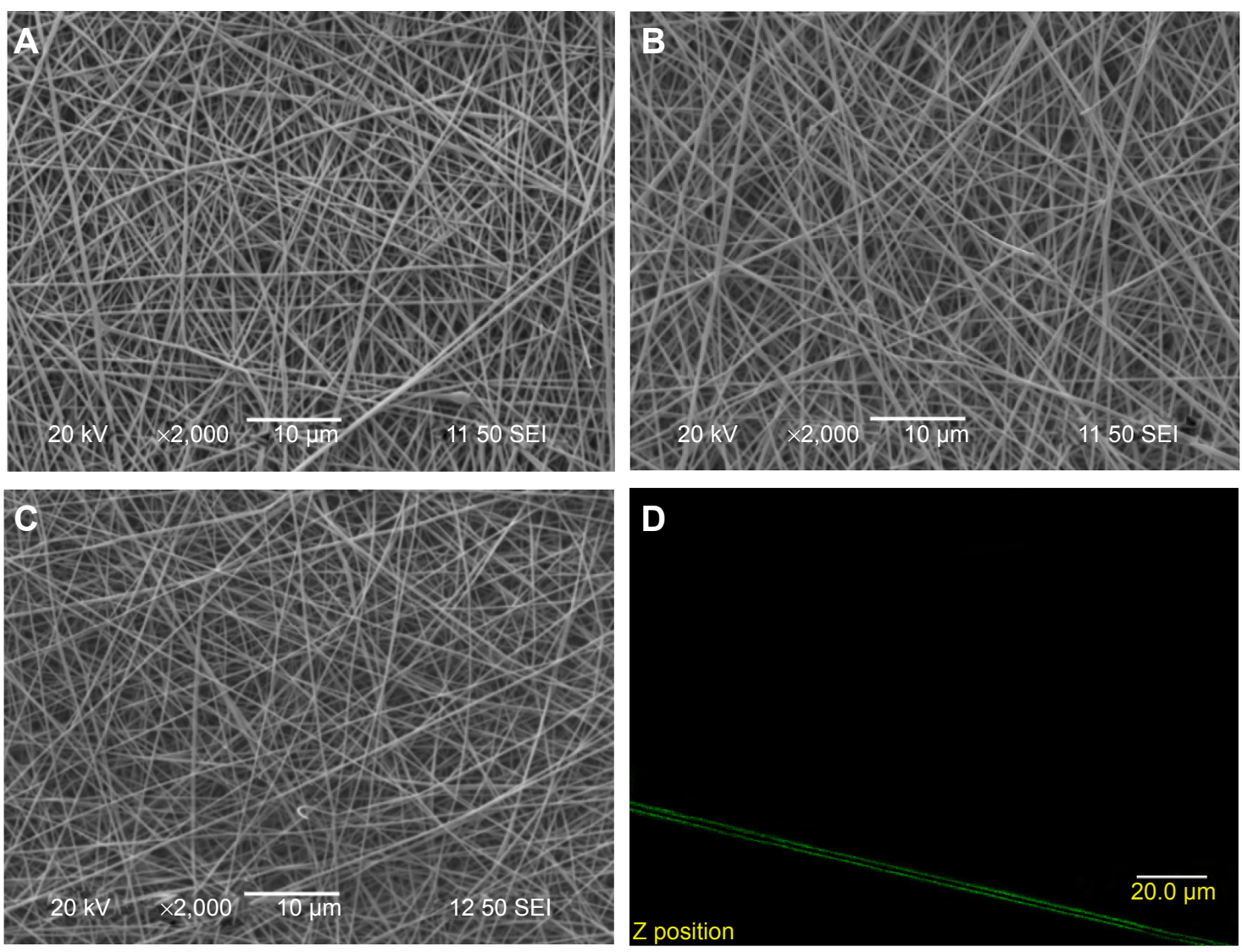

Figure 2 SEM images of PS (A), gelatin (B), and PS/gelatin nanofibrous membrane (C); confocal fluorescent image of FITC-gelatin/PS fiber (D).

Notes: Original magnifications: $\times 2,000(\mathbf{A}-\mathbf{C})$.

Abbreviations: FITC, fluorescein isothiocyanate; PS, polystyrene; SEM, scanning electron microscopy.

of the PS/gelatin did not present any significant changes compared to the spectrum of gelatin, indicating that PS has been completely covered by gelatin and thus no structural information on PS can be detected by ATR.
Contact angle measurement was used to evaluate surface hydrophobicity changes on the groups of PS, gelatin, and PS/gelatin. PS membrane is hydrophobic, with an average contact angle of $111.2^{\circ} \pm 0.04^{\circ}$, while water drops were

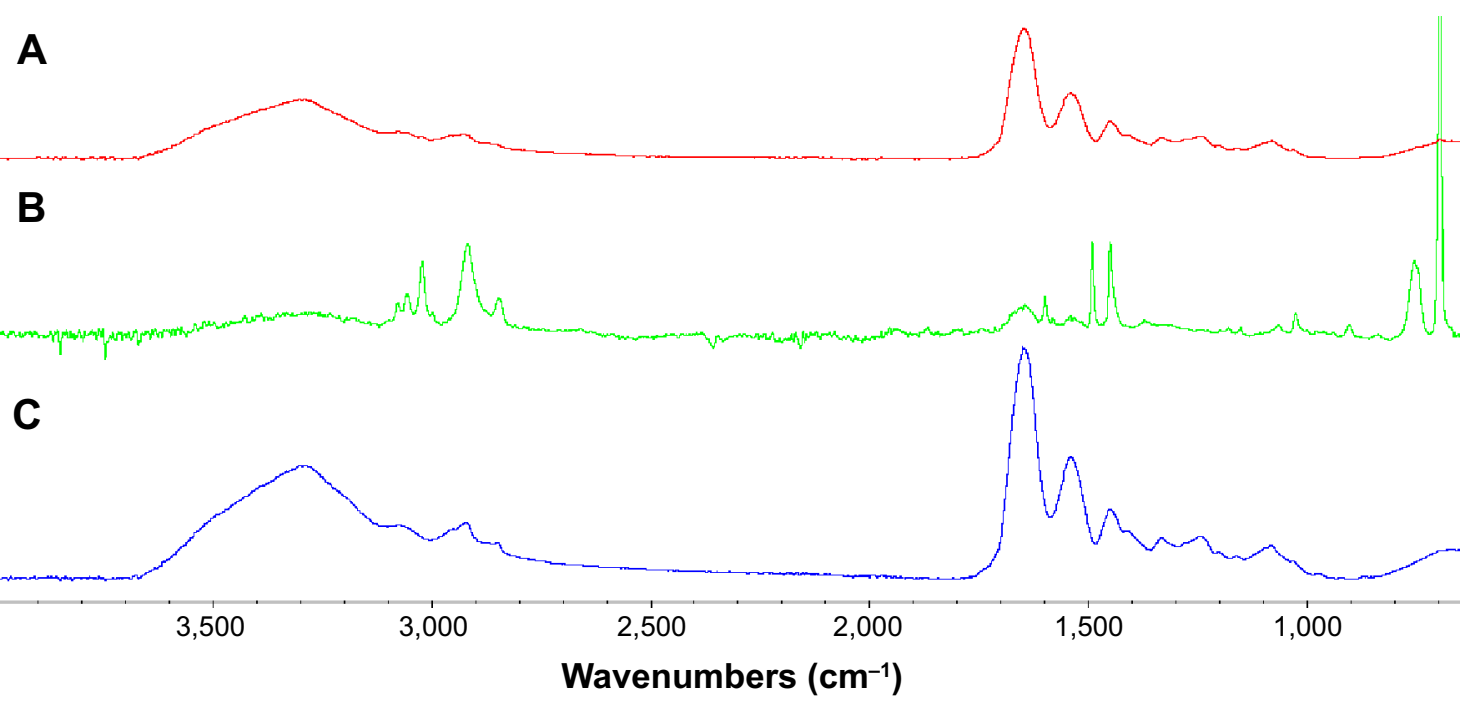

Figure 3 FTIR-ATR spectra of gelatin (A), polystyrene (B), and polystyrene-modified gelatin (C). Abbreviations: ATR, attenuated total reflection; FTIR, Fourier transform infrared spectroscopy. 
quickly absorbed in gelatin and PS/gelatin nanofibrous membranes.

An ideal pelvic patch should provide sufficient mechanical support to the pelvic floor and prevent pelvic organs from protruding into the vagina; meanwhile, the patch should not be too stiff so as to erode native tissues. ${ }^{29}$ In order to evaluate the mechanical properties of the PS/gelatin, loadextension measurements were performed with a strain rate of $5 \mathrm{~mm} / \mathrm{min}$. The Young's modulus of gelatin/PS scaffold is $12.42 \pm 2.92 \mathrm{MPa}$, similar to that of the corresponding native tissues. ${ }^{30}$

Overall, the gelatin sheath on PS nanofiber may promote cellular growth, being cell compatible while maintaining its good mechanical properties. ${ }^{31}$

\section{Cell adhesion on PS/gelatin nanofibrous mats}

The attached cells on the nanofibrous membrane were investigated under fluorescent microscopy after 24-hour culture. As shown in Figure 4, only a small amount of cells grew on the surface of the PS nanofibrous membrane, while adipose-derived stem cells (ADSCs) covered the full surface of the gelatin and PS/gelatin membranes. ADSCs show long spindle-shaped morphology on the surface of PS membrane, while the cells on PS nanofibrous membrane appeared more elongated and had a spindle-shaped morphology (Figure 4A) compared with those cultured in gelatin (Figure 4B) or PS/gelatin nanofibrous membrane (Figure 4C), which were more round and not elongated on the scaffold surface. Gelatin has long been known to provide good cell adhesion, with completely resorbable property in vivo and nonantigenicity under physiological conditions. ${ }^{32,33}$

\section{Host response to PS/gelatin nanofibrous membrane under vaginal submucosa}

To assess the host response in vivo, $\mathrm{PS} /$ gelatin nanofibrous membranes were implanted under the vaginal mucosa in rats, and the grafts were explanted with a small amount (approximately $2 \mathrm{~mm}$ ) of the surrounding native tissues for histological analysis at different postsurgery times, because it is a major concern regarding the medical device after implantation. ${ }^{34} \mathrm{H} \& \mathrm{E}$ staining (Figure 5) showed that a prominent host tissue response occurred in the 1 st and 2nd weeks, then decreased with time, and only minimal inflammatory responses were observed in the 6th week, with the evidence of the presence of multinucleated giant cells adjacent to materials. The results indicate that PS/gelatin nanofibrous patches triggered a much less severe inflammatory response, compared to that of PP (FDA-approved implant material) implantation in Liu et al's report. ${ }^{16}$ This may be attributed to the natural biomaterial coating that can reduce the immune reaction provoked by the synthetic materials. ${ }^{16,25,35}$

Futhermore, we detected T helper (Th) and macrophage (M1 and M2) reaction during implantation through immunochemical and gene expression analysis, because these cells can be characterized as Th1 or Th2 phenotype, M1 or M2 phenotype based on receptor expression and production of cytokines and effector molecules. These cells play critical roles in determining immune tolerance or immune rejection function. ${ }^{32,33,35,36}$ In general, M1-activated macrophages produce high levels of inducible nitric oxide synthetase (iNOS), secrete toxic reactive oxygen, and are inducer and effector cells in Th1-type inflammatory responses (cell-mediated rejection). M2-activated macrophages produce arginase (ARG) in the place of arginine, subsequently produce ornithine and polyamines, and are involved in polarized $\mathrm{Th} 2$ reactions to facilitate tissue repair and regeneration. ${ }^{36,37}$

The immunohistochemical evaluation showed that both Th1 cells (CXCR3 positive) and Th2 cells (CCR4 positive) were highly expressed in the first 2 weeks and then decreased with time (Figure 6). It was estimated that the Th1/Th2 cell ratio was $1.5,0.8,0.6,0.6$, and 0.9 from week 1 to week 6 , respectively. M1-(CCR7-positive) and M2 (CD163-positive)activated cells presented similar tendency (Figure 7), and
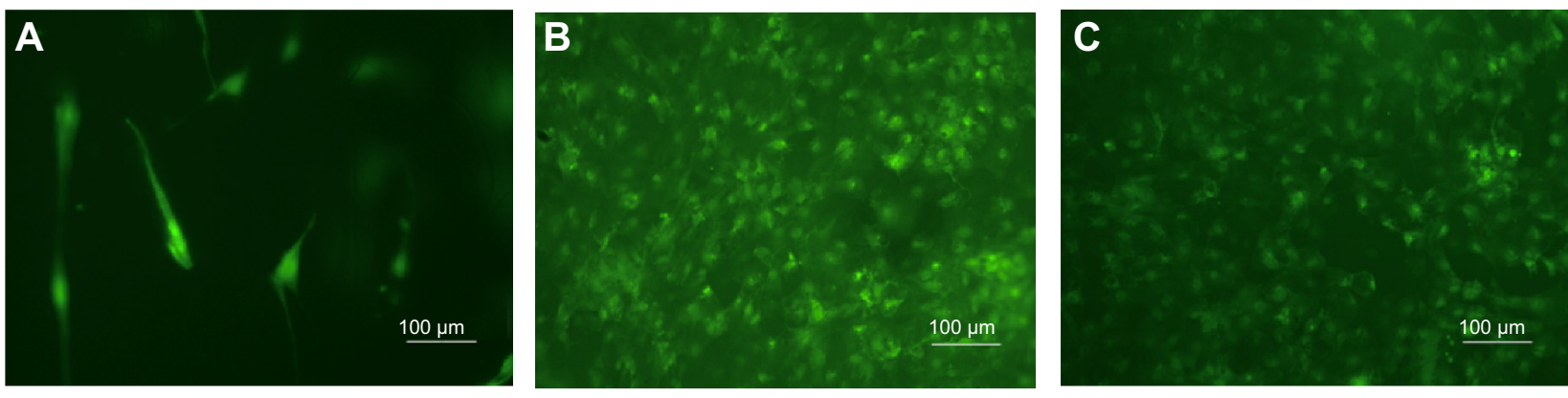

Figure 4 Cell growth on the surface of PS (A), gelatin (B), and PS/gelatin (C) nanofibrous membrane. Abbreviation: PS, polystyrene. 

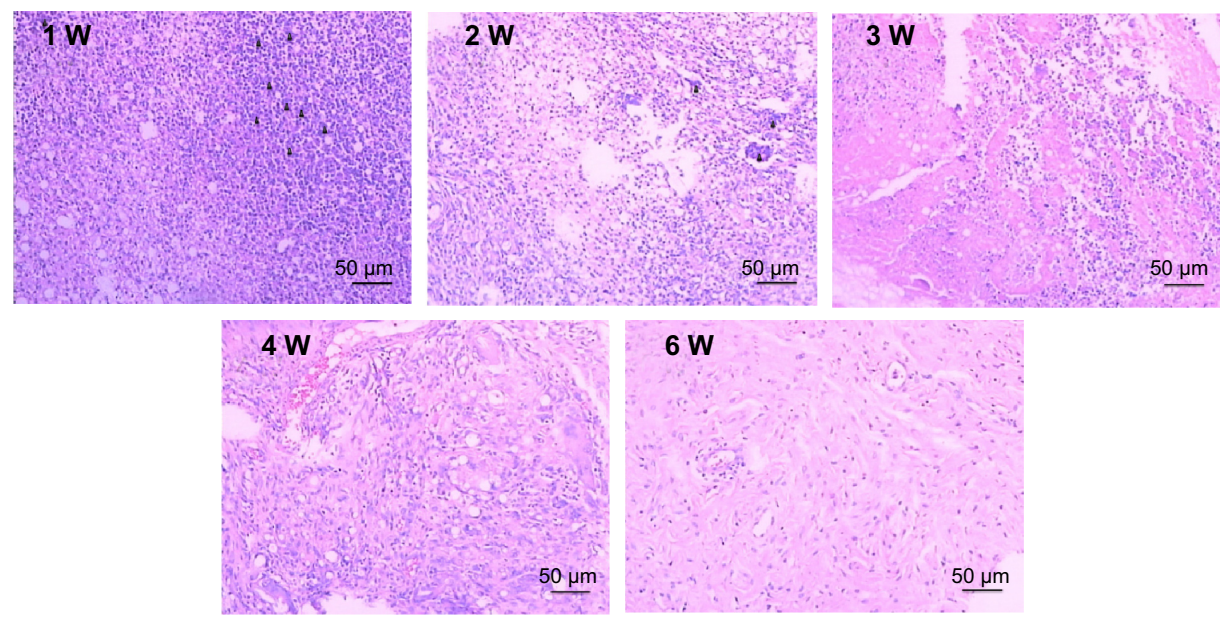

Figure 5 Inflammation responses to PS/gelatin at different time points postsurgery (black arrows: giant cells; scale bar $=50 \mu \mathrm{m}$ ). Notes: I W, 2 W, 3 W, 4 W, and $6 \mathrm{~W}$ indicate I week, 2 weeks, 3 weeks, 4 weeks, and 6 weeks, respectively. Abbreviation: PS, polystyrene.

the $\mathrm{M} 1 / \mathrm{M} 2$ ratio was $0.8,1.2,0.9,0.8$, and 1.1 at different postimplantation times, respectively. It is noticed that the ratios of Th1/Th2 and M1/M2 may be more important than the absolute number of cells because there was clearly a correlation of the Th1/Th2 and M1/M2 ratios to the remodeling outcome in previous studies. ${ }^{16,23}$

The results of gene expression correlated well with the results of the present study, with a predominantly Th2 cell and M2 macrophage response. The expression of interferon- $\gamma$ (IFN- $\gamma$, a Th1 marker) was high in the first 2 weeks (59.78and 68.33-fold increase, respectively), which then decreased to $37.24,28.25$, and 4.37 folds at 3 weeks, 4 weeks, and 6 weeks, while interleukin (IL)-10 (a Th2 marker) showed 40.7-, 210.74-, 172.34-, 101.92-, and 20.51-fold-increased expression at different postimplantation times (Figure 8). $i N O S$ (an M1 marker) and $A R G$ (an $\mathrm{M} 2$ marker) also showed results similar to that of immunohistochemical staining of polarized macrophages (Figure 7). These results suggest that gelatin coating of PS could evoke accommodation rather than immune rejection, which can be explained by the fact that the gelatin sheath on nanofibers provides an immune isolation for PS. Thus, the mat can combine the good biocompatibility of natural biomaterial (gelatin) after implantation and modulate the host response toward accommodation. ${ }^{16,38}$

There were several limitations in the present study. A limited number of surface markers were utilized for the characterization of the Th1/Th2 and M1/M2 phenotypes participating in the host response, due to the limited availability of antibodies specific for $\mathrm{T}$ helper cells and macrophage markers in the rat model. A limited number of gene expression markers were also used in this study. However, $I F N-\gamma, I L-10, i N O S$, and $A R G$ gene expressions are widely viewed as markers of Th1, Th2, M1, and M2 polarization, respectively.
CXCR3 (Th1)
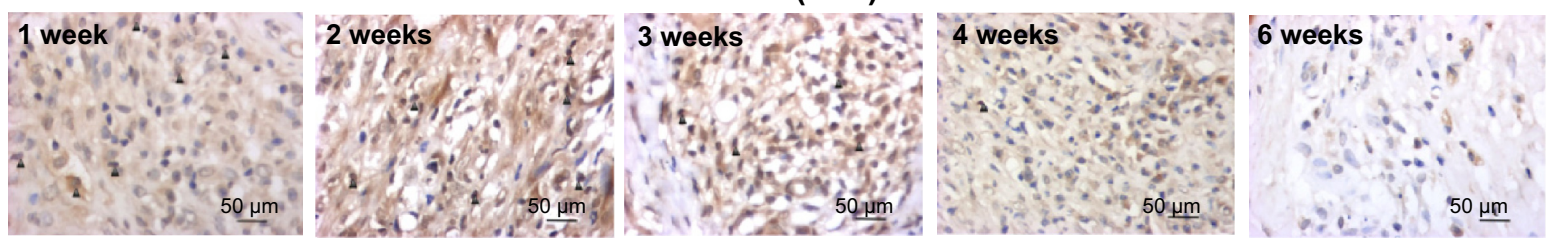

CCR4 (Th2)
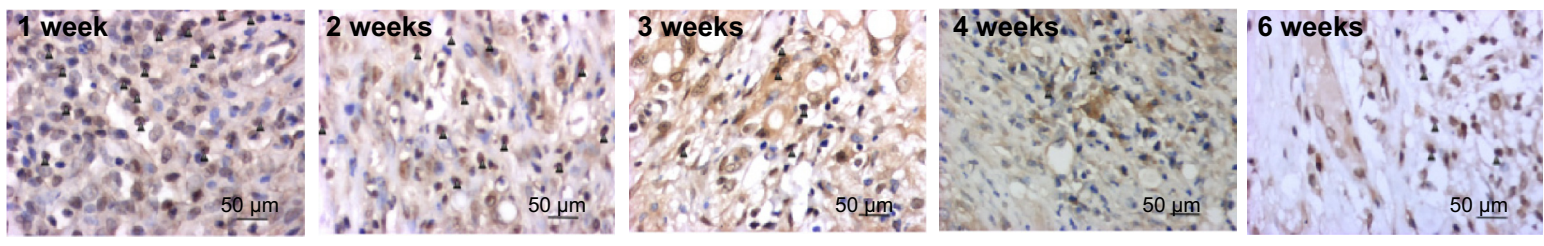

Figure 6 T-cell responses to PS/gelatin at different time points postsurgery (black arrows: positive cells).

Abbreviations: PS, polystyrene; Th, T helper cell. 


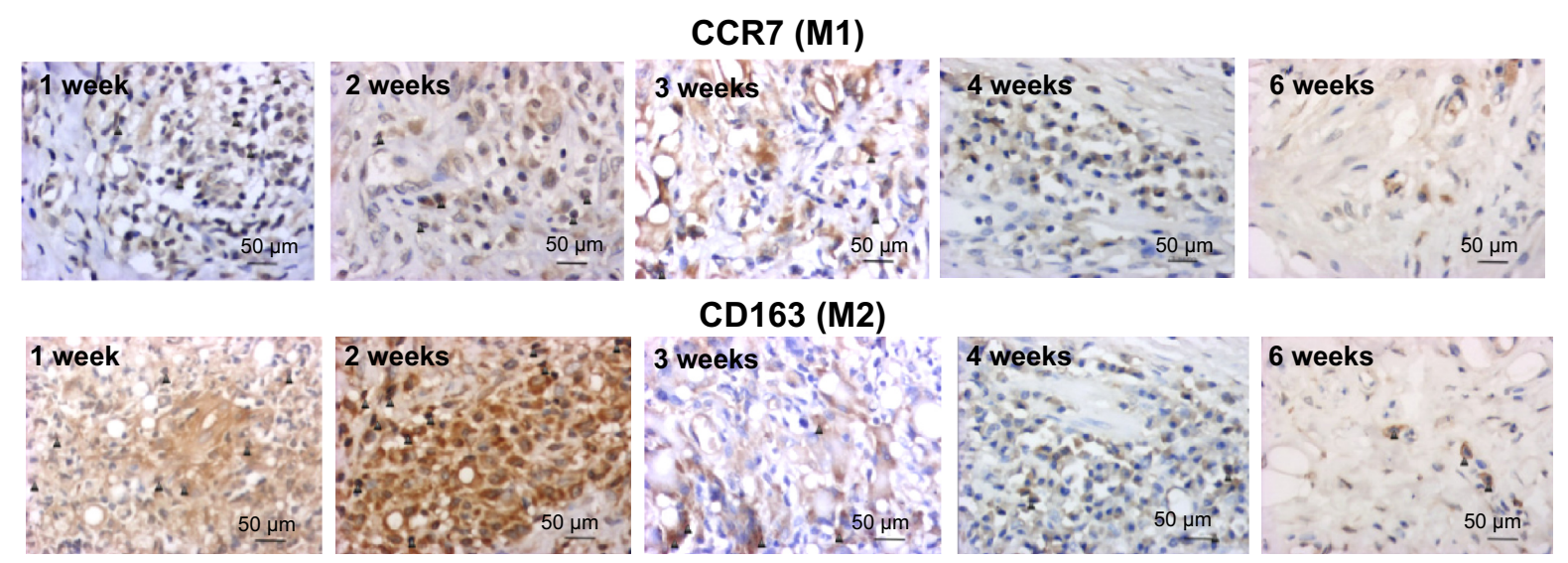

Figure 7 Macrophage cell responses to PS/gelatin at different time points postsurgery (black arrows: positive cells). Abbreviation: PS, polystyrene.

\section{Conclusion}

In this study, core-sheath PS/gelatin nanofibrous mats were fabricated for a novel application of pelvic reconstruction. The gelatin sheath provides the mat surface with hydrophilic property and enhances cell attachment, while the PS core provides the necessary mechanical strength. Furthermore, this device shows great graft accommodation in pelvic submucosa after implantation as observed from pathological examination and real-time polymerase chain reaction (real-time PCR). Thus, the core-sheath PS/gelatin mats are promising options in pelvic reconstruction.
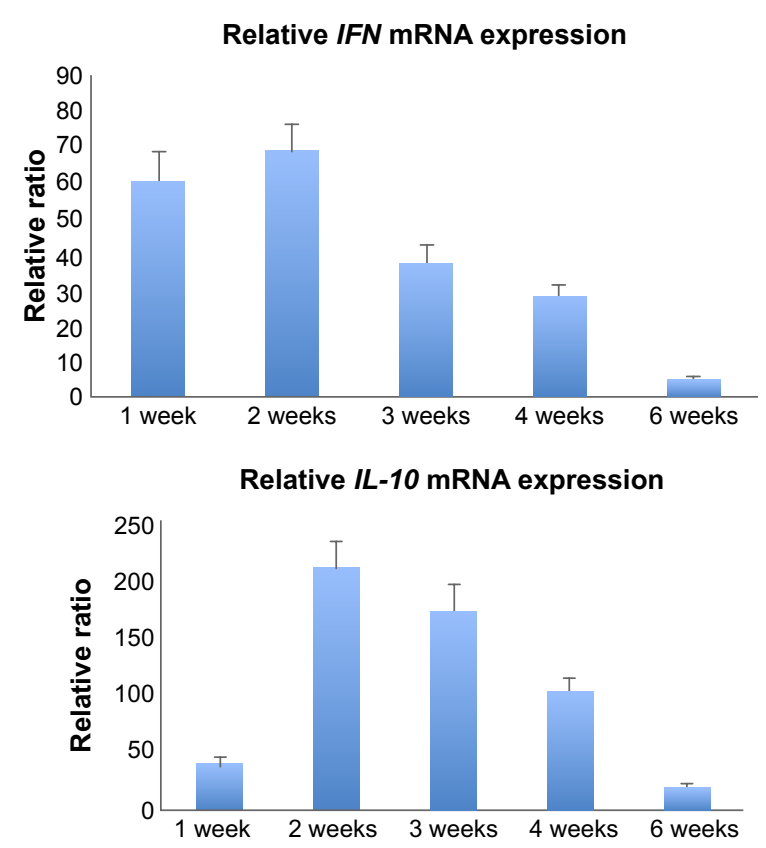

\section{Acknowledgments}

This work was supported by the International Science and Technology Cooperation Program of China (2013DFA31820), International Science and Technology Cooperation Program of Chongqing (CSTC2013gjhz80002), Chongqing Basic Scientific Research grant (cstc2013jcyjC80001), Chongqing Agriculture Development Grant (14408, 12402), China 863 Project (2014AA021602) NSERC, and CIHR Manitoba Partnership.

\section{Disclosure}

The authors report no conflicts of interest in this work.

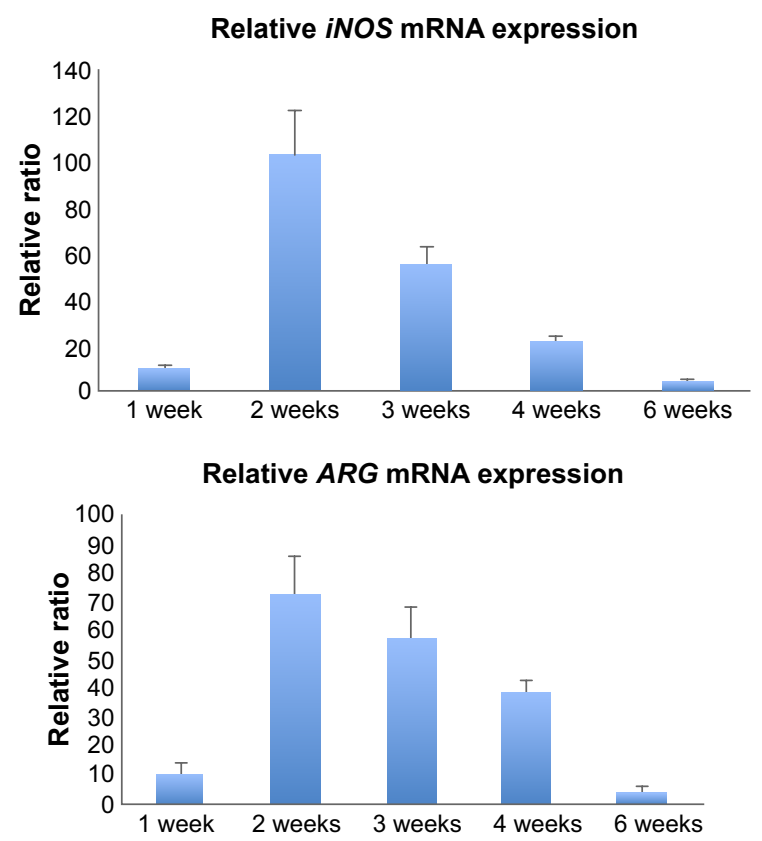

Figure 8 Gene expression of IFN- $\gamma$, iNOS, IL-I0, and ARG in explanted tissues at different time points. Abbreviations: IFN, interferon; iNOS, inducible nitric oxide synthase; IL, interleukin; ARG, arginase. 


\section{References}

1. Jelovsek JE, Maher C, Barber MD. Pelvic organ prolapse. Lancet. 2007;369(9566):1027-1038.

2. Jones KA, Shepherd JP, Oliphant SS, Wang L, Bunker CH, Lowder JL. Trends in inpatient prolapse procedures in the United States, 1979-2006. Am J Obstet Gynecol. 2010;202(5):e501-e507.

3. Olsen AL, Smith VJ, Bergstrom JO, Colling JC, Clark AL. Epidemiology of surgically managed pelvic organ prolapse and urinary incontinence. Obstet Gynecol. 1997;89(4):501-506.

4. Asante A, Whiteman MK, Kulkarni A, Cox S, Marchbanks PA, Jamieson DJ. Elective oophorectomy in the United States: trends and in-hospital complications, 1998-2006. Obstet Gynecol. 2010;116(5): 1088-1095.

5. Chaliha C, Khullar V. Surgical repair of vaginal prolapse: a gynaecological hernia. Int J Surg. 2006;4(4):242-250.

6. Altman D, Lopez A, Gustafsson C, Falconer C, Nordenstam J, Zetterstrom J. Anatomical outcome and quality of life following posterior vaginal wall prolapse repair using collagen xenograft. Int Urogynecol J Pelvic Floor Dysfunct. 2005;16(4):298-303.

7. Huebner M, Hsu Y, Fenner DE. The use of graft materials in vaginal pelvic floor surgery. Int J Gynaecol Obstet. 2006;92(3):279-288.

8. Griffis K, Hale DS. Grafts in pelvic reconstructive surgery. Clin Obstet Gynecol. 2005;48(3):713-723.

9. Nazemi TM, Kobashi KC. Complications of grafts used in female pelvic floor reconstruction: mesh erosion and extrusion. Indian J Urol. 2007; 23(2):153-160.

10. Liang CC, Lin YH, Chang YL, Chang SD. Urodynamic and clinical effects of transvaginal mesh repair for severe cystocele with and without urinary incontinence. Int J Gynaecol Obstet. 2011;112(3): $182-186$.

11. Iglesia CB, Sokol AI, Gutman RE, Sokol ER, Kudish BI, Shott S. Vaginal mesh for prolapse: a randomized controlled trial reply. Obstet Gynecol. 2010;116(6):1457-1457.

12. Rzepka J, Brocker K, Alt C, Corteville C, Sohn C, Lenz F. Pelvic organ prolapse: does the postoperative course of mesh-repair surgery differ in elderly women when compared with younger patients? J Obstet Gynaecol. 2010;30(8):852-856.

13. Trabuco EC, Klingele CJ, Gebhart JB. Xenograft use in reconstructive pelvic surgery: a review of the literature. Int Urogynecol J Pelvic Floor Dysfunct. 2007;18(5):555-563.

14. Chaliha C, Khalid U, Campagna L, Digesu GA, Ajay B, Khullar V. SIS graft for anterior vaginal wall prolapse repair - a case-controlled study. Int Urogynecol J Pelvic Floor Dysfunct. 2006;17(5): 492-497.

15. Badylak SF. The extracellular matrix as a biologic scaffold material. Biomaterials. 2007;28(25):3587-3593.

16. Liu L, Deng L, Wang Y, Ge L, Chen Y, Liang Z. Porcine urinary bladder matrix-polypropylene mesh: a novel scaffold material reduces immunorejection in rat pelvic surgery. Int Urogynecol J Pelvic Floor Dysfunct. 2012;23(9):1271-1278.

17. Ge LP, Zheng SQ, Wei H. Comparison of histological structure and biocompatibility between human acellular dermal matrix (ADM) and porcine ADM. Burns. 2009;35(1):46-50.

18. Franklin ME Jr, Gonzalez JJ Jr, Glass JL. Use of porcine small intestinal submucosa as a prosthetic device for laparoscopic repair of hernias in contaminated fields: 2-year follow-up. Hernia. 2004;8(3): 186-189.

International Journal of Nanomedicine

\section{Publish your work in this journal}

The International Journal of Nanomedicine is an international, peerreviewed journal focusing on the application of nanotechnology in diagnostics, therapeutics, and drug delivery systems throughout the biomedical field. This journal is indexed on PubMed Central,

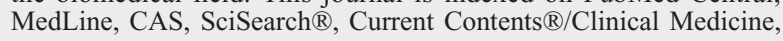

19. Chen J, Qiu X, Wang L, Zhong W, Kong J, Xing M. Free-Standing cell sheet assembled with ultrathin extracellular matrix as an innovative approach for biomimetic Tissues. Advanced Functional Materials. 2014;24(15):2216-2223.

20. Li Q, Wan W, Gao H et al. BMSCs laden injectable amino-diethoxypropane modified alginate-chitosan hydrogel for hyaline cartilage reconstruction. J Mater Chem B. In press 2014.

21. Li X, Zhou J, Liu Z, Chen J, Lü S, Sun H, et al. A PNIPAAm-based thermosensitive hydrogel containing SWCNTs for stem cell transplantation in myocardial repair. Biomaterials. 2014;35(22):5679-5688.

22. Ge L, Li Q, Huang Y, et al. Polydopamine-coated paper-stack nanofibrous membranes enhancing adipose stem cells' adhesion and osteogenic differentiation. J Mater Chem B. 2014;2(40):6917-6923.

23. Brown BN, Valentin JE, Stewart-Akers AM, McCabe GP, Badylak SF. Macrophage phenotype and remodeling outcomes in response to biologic scaffolds with and without a cellular component. Biomaterials. 2009;30(8):1482-1491.

24. Shi JB, Ouyang J, Li QT, et al. Cell-compatible hydrogels based on a multifunctional crosslinker with tunable stiffness for tissue engineering. J Mater Chem. 2012;22(45):23952-23962.

25. Shi J, Xing, Zhong W. Development of Hydrogels and Biomimetic Regulators as Tissue Engineering Scaffolds. Membranes. 2012;2(1):70-90.

26. Ingavle $\mathrm{G}$, Leach $\mathrm{JK}$. Advancements in electrospinning of polymeric nanofibrous scaffolds for tissue engineering. Tissue Eng Part B Rev. 2013;20(4):277-293.

27. Chen J, Zhou B, Li Q, et al. PLLA-PEG-TCH-labeled bioactive molecule nanofibers for tissue engineering. Int J Nanomedicine. 2011;6: 2533-2542.

28. Mohamed A, Xing MM. Nanomaterials and nanotechnology for skin tissue engineering. Int J Burns Trauma. 2012;2(1):29-41.

29. Mangera A, Bullock AJ, Roman S, Chapple CR, MacNeil S. Comparison of candidate scaffolds for tissue engineering for stress urinary incontinence and pelvic organ prolapse repair. BJU Int. 2013; 112(5):674-685.

30. Mangera A, Bullock AJ, Chapple CR, MacNeil S. Are biomechanical properties predictive of the success of prostheses used in stress urinary incontinence and pelvic organ prolapse? A systematic review. Neurourol Urodyn. 2012;31(1):13-21.

31. Ouasti S, Donno R, Cellesi F, Sherratt MJ, Terenghi G, Tirelli N. Network connectivity, mechanical properties and cell adhesion for hyaluronic acid/PEG hydrogels. Biomaterials. 2011;32(27):6456-6470.

32. Mano JF, Silva GA, Azevedo HS, et al. Natural origin biodegradable systems in tissue engineering and regenerative medicine: present status and some moving trends. J R Soc Interface. 2007;4(17):999-1030.

33. Cui YL, Hou X, Qi AD, et al. Biomimetic surface modification of poly (L-lactic acid) with gelatin and its effects on articular chondrocytes in vitro. J Biomed Mater Res A. 2003;66A(4):770-778.

34. Anderson JM, Rodriguez A, Chang DT. Foreign body reaction to biomaterials. Semin Immunol. 2008;20(2):86-100.

35. Meinel L, Hofmann S, Karageorgiou V, et al. The inflammatory responses to silk films in vitro and in vivo. Biomaterials. 2005;26(2):147-155.

36. Gordon S, Taylor PR. Monocyte and macrophage heterogeneity. Nat Rev Immunol. 2005;5(12):953-964.

37. Mosser DM. The many faces of macrophage activation. J Leukoc Biol. 2003;73(2):209-212.

38. Badylak SE, Gilbert TW. Immune response to biologic scaffold materials. Semin Immunol. 2008;20(2):109-116.

Journal Citation Reports/Science Edition, EMBase, Scopus and the Elsevier Bibliographic databases. The manuscript management system is completely online and includes a very quick and fair peer-review system, which is all easy to use. Visit http://www.dovepress.com/ testimonials.php to read real quotes from published authors. 